\title{
The effect of body position on pulmonary function: a systematic review
}

Shikma Katz ${ }^{1,3+}$, Nissim Arish ${ }^{2,4 \dagger}$, Ariel Rokach ${ }^{2,4^{*}}$, Yacov Zaltzman ${ }^{1}$ and Esther-Lee Marcus ${ }^{1,4}$

\begin{abstract}
Background: Pulmonary function tests (PFTs) are routinely performed in the upright position due to measurement devices and patient comfort. This systematic review investigated the influence of body position on lung function in healthy persons and specific patient groups.

Methods: A search to identify English-language papers published from 1/1998-12/2017 was conducted using MEDLINE and Google Scholar with key words: body position, lung function, lung mechanics, lung volume, position change, positioning, posture, pulmonary function testing, sitting, standing, supine, ventilation, and ventilatory change. Studies that were quasi-experimental, pre-post intervention; compared $\geq 2$ positions, including sitting or standing; and assessed lung function in non-mechanically ventilated subjects aged $\geq 18$ years were included. Primary outcome measures were forced expiratory volume in $1 \mathrm{~s}$ (FEV1), forced vital capacity (FVC, FEV1/FVC), vital capacity (VC), functional residual capacity (FRC), maximal expiratory pressure (PEmax), maximal inspiratory pressure (PImax), peak expiratory flow (PEF), total lung capacity (TLC), residual volume (RV), and diffusing capacity of the lungs for carbon monoxide (DLCO). Standing, sitting, supine, and right- and left-side lying positions were studied.

Results: Forty-three studies met inclusion criteria. The study populations included healthy subjects (29 studies), lung disease (nine), heart disease (four), spinal cord injury (SCl, seven), neuromuscular diseases (three), and obesity (four). In most studies involving healthy subjects or patients with lung, heart, neuromuscular disease, or obesity, FEV1, FVC, FRC, PEmax, PImax, and/or PEF values were higher in more erect positions. For subjects with tetraplegic SCl, FVC and FEV1 were higher in supine vs. sitting. In healthy subjects, DLCO was higher in the supine vs. sitting, and in sitting vs. side-lying positions. In patients with chronic heart failure, the effect of position on DLCO varied.

Conclusions: Body position influences the results of PFTs, but the optimal position and magnitude of the benefit varies between study populations. PFTs are routinely performed in the sitting position. We recommend the supine position should be considered in addition to sitting for PFTs in patients with $\mathrm{SCl}$ and neuromuscular disease. When treating patients with heart, lung, $\mathrm{SCl}$, neuromuscular disease, or obesity, one should take into consideration that pulmonary physiology and function are influenced by body position.
\end{abstract}

Keywords: Body position, Lung volume, Physical therapy, Positioning, Posture, Pulmonary function, Sitting, Supine, Standing

\section{Background}

Pulmonary function tests (PFTs) provide objective, quantifiable measures of lung function. They are used to evaluate and monitor diseases that affect heart and lung function, to monitor the effects of environmental,

\footnotetext{
* Correspondence: rokach.ariel@gmail.com

†Shikma Katz and Nissim Arish contributed equally to this work.

${ }^{2}$ Pulmonary Institute, Shaare Zedek Medical Center, POB 3235, Jerusalem, Israel

${ }^{4}$ Hebrew University-Hadassah Faculty of Medicine, Jerusalem, Israel Full list of author information is available at the end of the article
}

occupational, and drug exposures, to assess risks of surgery, and to assist in evaluations performed before employment or for insurance purposes. Spirometric examination is the most common form of PFT [1]. According to ATS/ERS guidelines, PFTs may be performed either in the sitting or standing position, and the position should be recorded on the report. Sitting is preferable for safety reasons to avoid falling due to syncope [2], and might also be more convenient because of the measurement devices and patient comfort. However, people who

(c) The Author(s). 2018 Open Access This article is distributed under the terms of the Creative Commons Attribution 4.0 International License (http://creativecommons.org/licenses/by/4.0/), which permits unrestricted use, distribution, and reproduction in any medium, provided you give appropriate credit to the original author(s) and the source, provide a link to the Creative Commons license, and indicate if changes were made. The Creative Commons Public Domain Dedication waiver (http://creativecommons.org/publicdomain/zero/1.0/) applies to the data made available in this article, unless otherwise stated. 
suffer from neuromuscular disease, morbid obesity, and other conditions may find it difficult to sit or stand during this test, which may influence their results.

One of the main goals of positioning, and specifically the use of upright positions, is to improve lung function in patients with respiratory disorders, heart failure, neuromuscular disease, spinal cord injury (SCI), and obesity, and in the past 20 years, various studies regarding the influence of body position on respiratory mechanics and/or function have been published. However, we did not find a systematic review that integrates findings from studies involving non-mechanically ventilated adults to derive clinical implications for respiratory care and pulmonary function test (PFT) execution.

We aimed to systematically review studies that evaluated the effect of body position on lung function in healthy subjects and non-mechanically ventilated patients with lung disease, heart disease, SCI, neuromuscular disease, and obesity.

\section{Methods}

Two researchers (SK., E-LM.) searched MEDLINE and Google Scholar for studies published from January 1998December 2017 using the key words body position, lung function, lung mechanics, lung volumes, position change, positioning, posture, PFTs, sitting, standing, supine, ventilation, and ventilatory change, in various combinations. Each search term combination included at least one key word related to pulmonary function and at least one related to body position. The year 1998 was chosen as the beginning point due to the publication of the seminal study by Meysman and Vincken [3]. A total of $972 \mathrm{ab-}$ stracts identified in the search were screened by the same two researchers, and full text of 151 potentially relevant articles was obtained. The full texts were evaluated and categorized, and 108 articles not fulfilling the inclusion criteria were excluded (Fig. 1).

Articles were included if they met the following criteria: (1) Quasi-experimental, pre-post intervention. (2) Two or more body positions compared, including at least the sitting or standing position. (3) Outcome measures included assessment of lung function by forced vital capacity (FVC), forced expiratory volume in $1 \mathrm{~s}$ (FEV1), FEV1/ FVC, vital capacity (VC), functional residual capacity (FRC), maximal expiratory pressure (PEmax), maximal inspiratory pressure (PImax), peak expiratory flow (PEF), total lung capacity (TLC), residual volume (RV), or diffusing capacity of the lungs for carbon monoxide (DLCO). (4) Study population of non-mechanically ventilated subjects. (5) Participants aged $\geq 18$ years. (6) English language. Studies assessing lung function using other criteria and those without statistical comparisons of lung function in different positions, those enrolling individuals $<18$ years or on mechanical ventilation, published conference abstracts, and systematic reviews were excluded.

\section{Positions studied}

1. Standing - unsupported active standing

2. Sitting - sitting on a chair or wheelchair with the backrest at $90^{\circ}$ and all limbs supported

3. Supine - lying flat on the back

4. Right-side lying (RSL) - lying straight on the right side

5. Left-side lying (LSL) - lying straight on the left side

Outcome measures and defined thresholds for clinical significance

1. FVC - forced vital capacity

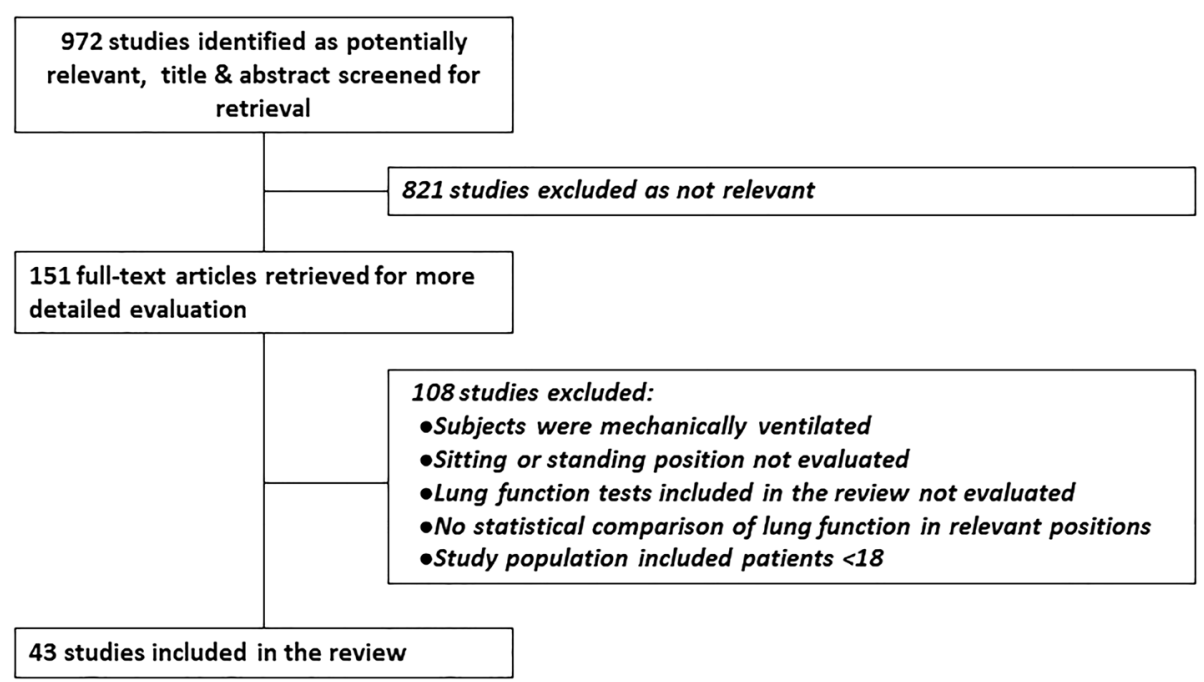

Fig. 1 Study flow diagram 
- Change of $200 \mathrm{ml}$ or $12 \%$ from baseline values in FVC [4]

2. FEV1- forced expiratory volume in $1 \mathrm{~s}$

- Change of $200 \mathrm{ml}$ or $12 \%$ from baseline values in FEV1 [4]

3. FEV1/FVC - forced expiratory volume in $1 \mathrm{~s}$ divided by forced vital capacity

- FEV1/FVC $<0.7$ is defined as obstructive disease

4. VC - vital capacity

5. FRC - functional residual capacity

- Change $>10 \%[5]$

6. TLC - total lung capacity

- Change $>10 \%[5]$

7. RV - residual volume

8. Maximal expiratory pressure (PEmax)

- Change $\geq 24 \mathrm{cmH} 2 \mathrm{O}$ [6-8]

9. Maximal inspiratory pressure (PImax)

- Change $\leq-13 \mathrm{cmH} 2 \mathrm{O}[6-8]$

10. Peak expiratory flow (PEF)

- Change $>10 \%$ or $60 \mathrm{~L} / \mathrm{min}[9,10]$

11. Diffusing capacity of the lungs for carbon monoxide (DLCO)

- Change $\geq 10 \%$ in DLCO $[11,12]$

Two experienced pulmonologists (NA, AR) reviewed the included studies in consensus to identify statistically significant and clinically important differences in pulmonary function. Results from articles included in the review were evaluated by all authors and categorized by study population, body positions studied, and outcome measures. Data from included studies was extracted by four authors (NA, AR, SK, E-LM.) independently and in consultation when questions arose. The review was performed according to the PRISMA guidelines [13].

Although these are not interventional studies, strictly speaking, we have chosen to assess them as "before and after intervention," wherein the posture/ position change is the maneuver of interest. Level of evidence was assessed according to the American Academy of Neurology (AAN) Classification of Evidence for therapeutic intervention [14]. Risk of bias was assessed according to the Quality Assessment Tool for Before-After (Pre-Post) Studies with No Control Group developed by the National Heart, Lung and Blood Institute (NHLBI) of the US National Institutes of Health (NIH) [15]. This tool is comprised of 12 questions assessing various aspects of the quality of the study. Two authors (E-LM, SK) independently scored each study using the technique from Kunstler et al. [16]. Differences were resolved in consensus, in consultation with a third author (YZ). The risk of bias was categorized as low (score 76-100\%), moderate $(26-75 \%)$ or high $(0-25 \%)$.

\section{Results}

Studies included in the review

A total of 43 studies fully met inclusion criteria and were included in the review (Fig. 1). All studies used either consecutive, convenience, or volunteer sampling to enroll healthy individuals or subjects with various medical conditions. All studies provide Class III level of evidence.

The protocols and level of bias in the various studies are shown in Table 1 and Additional file 1: Table S1. Risk of bias was assessed as moderate in 41 studies and low in two. Quality issues were primarily related to sampling techniques for enrolling study participants. All studies used non-random sampling. Some studies investigating healthy subjects included convenience samples of young participants, mainly students. Only 7/43 studies reported sample size calculations required to reach statistical power. In addition, the details of the intervention protocol were not clearly reported in some studies (Table 1) and due to the nature of the study assessors could not be blinded to patient position or outcomes from previous tests.

A summary of study characteristics, including the positions studied, outcome measures, and main results according to the study population, is shown in Table 2 . Out of 43 studies, 29 included healthy subjects, nine included patients with lung disease, four included patients with heart disease, seven included patients with SCI, three included patients with neuromuscular diseases, and four included patients with obesity. Additional file 2: Table S2 summarizes only the statistically significant findings for each relevant outcome variable, according to position, for each of the populations studied.

\section{FVC}

The association between FVC and body position in healthy subjects was investigated in 13 studies $[3,17-$ 28]. There was a clinical and statistically significant increase in FVC in sitting vs. supine positions [3, 18, 2227], in sitting vs. RSL and LSL [3, 21], standing vs. supine [19, 23], and standing vs. RSL and LSL [19]. In a smaller number of studies there was no change between standing and sitting [19], sitting and supine [17, 21, 28] or sitting and RSL or LSL [21], and one study [22] found a decrease in FVC from sitting to standing that was statistically but not clinically significant. Thus, in the majority of studies the more upright position was associated with increased FVC.

Four studies included subjects with lung disease [2932]. Among asthmatic patients in one study FVC increased significantly from supine to standing [30]; however, there was no significant difference between standing and sitting or between sitting and supine, RSL, or LSL. Another study reported a statistically and 
Table 1 Study protocols

\begin{tabular}{|c|c|c|c|c|}
\hline 1st Author (year) & Procedure & $\begin{array}{l}\text { Posture and Test } \\
\text { Randomization }\end{array}$ & $\begin{array}{l}\text { Adjustment period to posture } \\
\text { prior to measurement }\end{array}$ & $\begin{array}{l}\text { Risk of } \\
\text { Bias }\end{array}$ \\
\hline Antunes (2016) [45] & $\begin{array}{l}\text { Mini Wright }{ }^{\oplus} \text { (Clement Clarke } \\
\text { International Ltd. Edinburgh } \\
\text { Way Harlow, Essex, UK) peak flow meter } \\
\text { portable device with a } \\
\text { disposable mouthpiece }\end{array}$ & Random position order & $1 \mathrm{~min}$ & Moderate \\
\hline Badr (2002) [46] & $\begin{array}{l}\text { Pressure manometer, vitalograph } \\
\text { (Compact, Vitalograph Ltd., } \\
\text { Buckingham, UK) }\end{array}$ & $\begin{array}{l}\text { Random position order } \\
\text { Random test order (PEF and } \\
\text { PEmax) Subjects instructed } \\
\text { on equipment use, practiced } \\
\text { before test }\end{array}$ & $5 \mathrm{~min}$ & Low \\
\hline Baydur (2001) [35] & Spirometry & Random position order & N/A & Moderate \\
\hline Ben-Dov (2009) [17] & Spirometry & N/A & N/A & Moderate \\
\hline Benedik (2009) [52] & Helium dilution & First position always sitting & $5 \mathrm{~min}$ & Moderate \\
\hline Ceridon (2011) [18] & $\begin{array}{l}\text { Spirometry, DLCO measured } \\
\text { by rebreathe technique }\end{array}$ & N/A & $\begin{array}{l}30 \text { min supine position prior } \\
\text { to test Time prior to seated } \\
\text { measurement not mentioned }\end{array}$ & Moderate \\
\hline Chang (2005) [53] & $\begin{array}{l}\text { Spirometry, FRC measured } \\
\text { using helium dilution }\end{array}$ & First position always supine & $5 \mathrm{~min}$ & Moderate \\
\hline Costa (2015) [54] & Mouth pressure meter & Random position order & $10 \mathrm{~min}$ & Moderate \\
\hline De (2012) [29] & Spirometry & $\begin{array}{l}\text { First position was always } \\
\text { sitting }\end{array}$ & N/A & Moderate \\
\hline Elkins (2005) [47] & $\begin{array}{l}\text { Pressure manometer, spirometry - mass flow } \\
\text { sensor }\end{array}$ & $\begin{array}{l}\text { Random position and test } \\
\text { order (PEF, PEmax) } \\
\text { Subjects instructed } \\
\text { on equipment use, practiced } \\
\text { before test }\end{array}$ & $5 \mathrm{~min}$ & Low \\
\hline Faggiano (1998) [58] & $\begin{array}{l}\text { Single breath technique using } \\
\text { a Medical Graphics PF/DX } \\
\text { module (Medical Graphics } \\
\text { St. Paul, Minn, USA) for determining DLCO }\end{array}$ & Random position order & $10 \mathrm{~min}$ & Moderate \\
\hline Ganapathi (2015) [19] & $\begin{array}{l}\text { Digital spirometry (BIOPAC System } \\
\text { Inc. Goleta, California, USA) }\end{array}$ & N/A & N/A & Moderate \\
\hline Gianinis (2013) [48] & $\begin{array}{l}\text { Portable peak expiratory } \\
\text { flow-device }\end{array}$ & Random position order & N/A & Moderate \\
\hline $\operatorname{Kim}(2012)$ [36] & Spirometry & N/A & N/A & Moderate \\
\hline Linn (2000) [33] & Spirometry & Random position order & N/A & Moderate \\
\hline Manning (1999) [20] & $\begin{array}{l}\text { Spirometry, single breath for determining } \\
\text { DLCO }\end{array}$ & $\begin{array}{l}\text { Two protocols (Session A \& } \\
\text { B). First chosen at random } \\
\text { then alternated for } \\
\text { successive subjects. } \\
\text { First position always sitting. }\end{array}$ & $15 \min$ & Moderate \\
\hline McCoy (2010) [49] & Peak flow meter & $\begin{array}{l}\text { Random position order. } \\
\text { Subjects instructed on } \\
\text { equipment use, practiced } \\
\text { before test }\end{array}$ & N/A & Moderate \\
\hline Melam (2014) [30] & $\begin{array}{l}\text { Spirometry (Excel/PC-based } \\
\text { pulmonary function tests) }\end{array}$ & Random position order & N/A & Moderate \\
\hline Meysman (1998) [3] & Spirometry, peak flow meter & Random position order & $10 \mathrm{~min}$ & Moderate \\
\hline Miccinilli (2016) [40] & Spirometry & N/A & N/A & Moderate \\
\hline Mohammed (2017) [31] & Spirometry & $\begin{array}{l}\text { Order of positions always } \\
\text { standing, sitting, supine, } \\
\text { lateral decubitus }\end{array}$ & N/A & Moderate \\
\hline Myint (2017) [42] & Spirometry & $\begin{array}{l}\text { Order of positions was } \\
\text { standing, sitting, supine }\end{array}$ & N/A & Moderate \\
\hline Naitoh (2014) [39] & Spirometry, breath dynamometer (Chest Co. Ltd) & First position always sitting & N/A & Moderate \\
\hline
\end{tabular}


Table 1 Study protocols (Continued)

\begin{tabular}{|c|c|c|c|c|}
\hline 1st Author (year) & Procedure & $\begin{array}{l}\text { Posture and Test } \\
\text { Randomization }\end{array}$ & $\begin{array}{l}\text { Adjustment period to posture } \\
\text { prior to measurement }\end{array}$ & $\begin{array}{l}\text { Risk of } \\
\text { Bias }\end{array}$ \\
\hline Ogiwara (2002) [55] & $\begin{array}{l}\text { Vitalpower KH-101 (Chest M.I. } \\
\text { Inc., Japan) }\end{array}$ & Random position order & $10 \mathrm{~min}$ & Moderate \\
\hline Ottaviano (2016) [50] & Peak flow meter & Random position order & N/A & Moderate \\
\hline Palermo (2005) [21] & $\begin{array}{l}\text { Spirometry, DLCO measured by a } \\
\text { single breath technique }\end{array}$ & Random position order & $15 \mathrm{~min}$ & Moderate \\
\hline Park (2010) [34] & Spirometry & N/A & N/A & Moderate \\
\hline Patel (2015) [22] & Spirometry & First position always sitting & N/A & Moderate \\
\hline Peces-Barba (2004) [56] & $\begin{array}{l}\text { Single breath technique, } \\
\text { rebreathing technique for determining DLCO }\end{array}$ & N/A & $3-5 \min$ & Moderate \\
\hline Poussel (2014) [38] & Spirometry & Random position order & N/A & Moderate \\
\hline Razi (2007) [32] & Spirometry & Alternately sitting, standing & $\begin{array}{l}\text { N/A } 15 \text { min between } \\
\text { positions }\end{array}$ & Moderate \\
\hline Roychowdhury (2011) [44] & Spirometry & N/A & $\begin{array}{l}\mathrm{N} / \mathrm{A} \\
5 \text { min rest between positions }\end{array}$ & Moderate \\
\hline Saxena (2006) [23] & Spirometry & N/A & N/A & Moderate \\
\hline Sebbane (2015) [41] & $\begin{array}{l}\text { Spirometry, multiple breath } \\
\text { helium dilution method }\end{array}$ & First position always sitting & N/A & Moderate \\
\hline Stewart (2000) [24] & Single breath method for determining DLCO & $\mathrm{N} / \mathrm{A}, 72 \mathrm{~h}$ between positions & $15 \min$ & Moderate \\
\hline $\begin{array}{l}\text { Terson de Paleville (2014) } \\
\text { [37] }\end{array}$ & $\begin{array}{l}\text { Spirometry, MP45-36-350 } \\
\text { differential pressure transducer } \\
\text { Validyne Engineering, } \\
\text { (Northridge Ca, USA) }\end{array}$ & First position always sitting & $30 \mathrm{~min}$ & Moderate \\
\hline Terzano (2009) [57] & Single breath DLCO technique & Random position order & At least $15 \mathrm{~min}$ & Moderate \\
\hline Tsubaki (2009) [28] & $\begin{array}{l}\text { Micro RPM } 01 \text { (Micro Medical, UK), } \\
\text { spirometry }\end{array}$ & Random position order & N/A & Moderate \\
\hline Varrato (2001) [25] & Spirometry & N/A & N/A & Moderate \\
\hline Vilke (2000) [26] & Spirometry & $\begin{array}{l}\text { First position always supine/ } \\
\text { prone }\end{array}$ & N/A & Moderate \\
\hline Wallace (2013) [51] & Peak flow meter & Random position order & N/A & Moderate \\
\hline Watson (2005) [43] & $\begin{array}{l}\text { Multi-breath helium dilution, } \\
\text { spirometry }\end{array}$ & N/A & N/A & Moderate \\
\hline Yap (2000) [27] & $\begin{array}{l}\text { Spirometry, FRC was measured } \\
\text { using helium dilution }\end{array}$ & First position always sitting & $5 \mathrm{~min}$ & Moderate \\
\hline
\end{tabular}

Risk of bias was assessed using the Quality Assessment Tool for Before-After (Pre-Post) Studies with No Control Group [15, 16]

$D L C O$ Diffusing capacity of the lungs for carbon monoxide, FRC Functional residual capacity

$N / A$ Not available, not reported in the study

clinically significant increase in FVC in standing vs. sitting, supine, RSL, and LSL and in sitting vs. supine, RSL and LSL [31]. Among obese asthmatic patients [32], and among patients with chronic obstructive pulmonary disease (COPD) [29], no difference was found in FVC between standing and sitting.

Three studies included subjects with congestive heart failure (CHF) [18, 21, 27]. In one study, FVC was reported $200 \mathrm{ml}$ higher in sitting vs. RSL and LSL [21], and in the other two studies FVC was higher in sitting vs. supine by $350-400 \mathrm{ml}$, which has clinical significance $[18,27]$.
Six studies included patients with SCI [17, 33-37]. The effect of body position on FVC depends on the level and extent of injury. Among those with cervical SCI, FVC was higher in the supine vs. sitting position $[17,33$, 34]. Other studies [35-37] did not find significant differences in FVC for patients with SCI in a pooled group of all levels of injury for these positions. However, in patients with cervical SCI, as well as those with thoracic injury in one study [36], there was an increased FVC in the supine vs. sitting, while in those with thoracic or lumbar injury FVC was higher in the sitting position [37]. The differences did not always reach statistical 


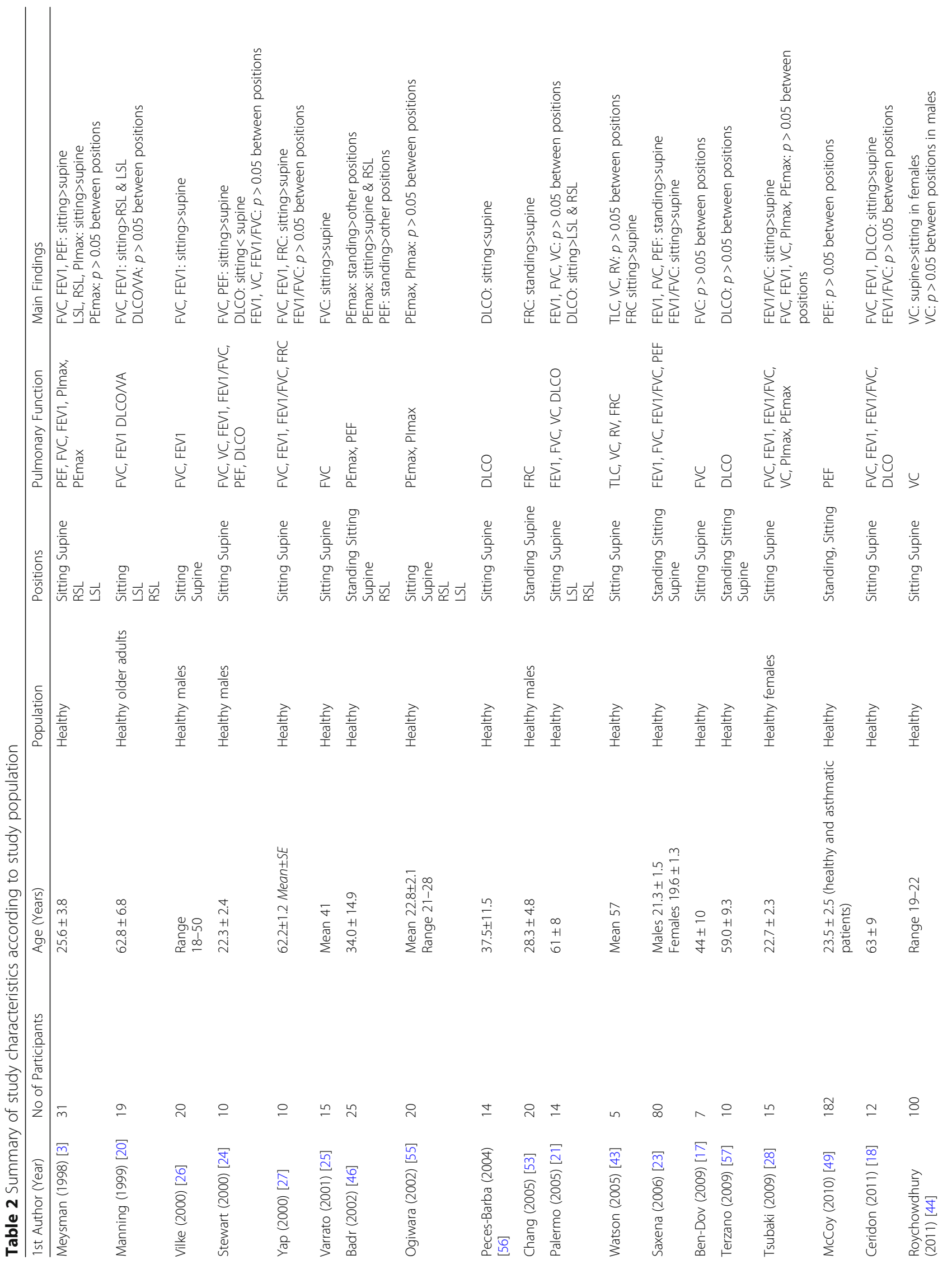




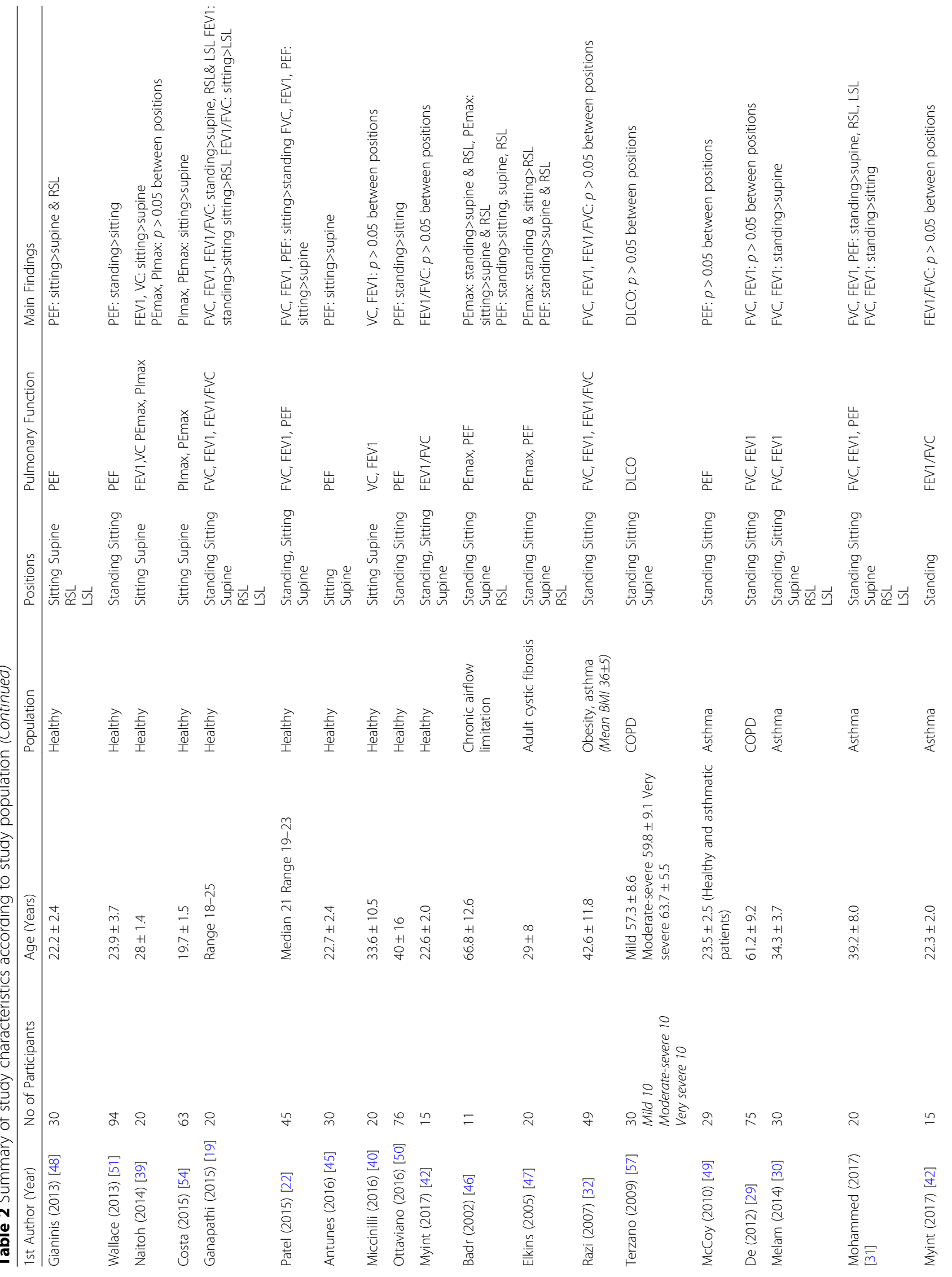




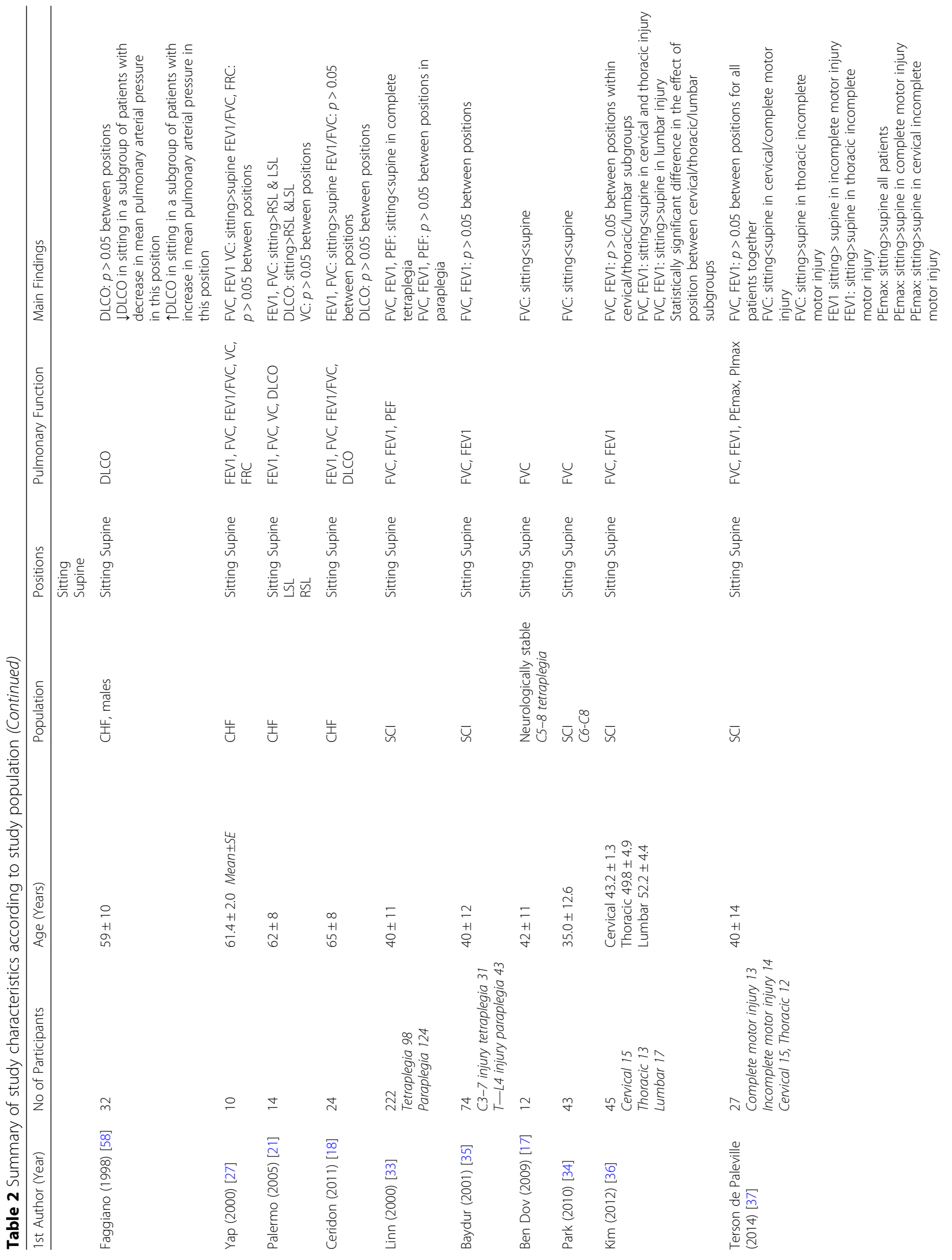


Katz et al. BMC Pulmonary Medicine (2018) 18:159

Page 9 of 16

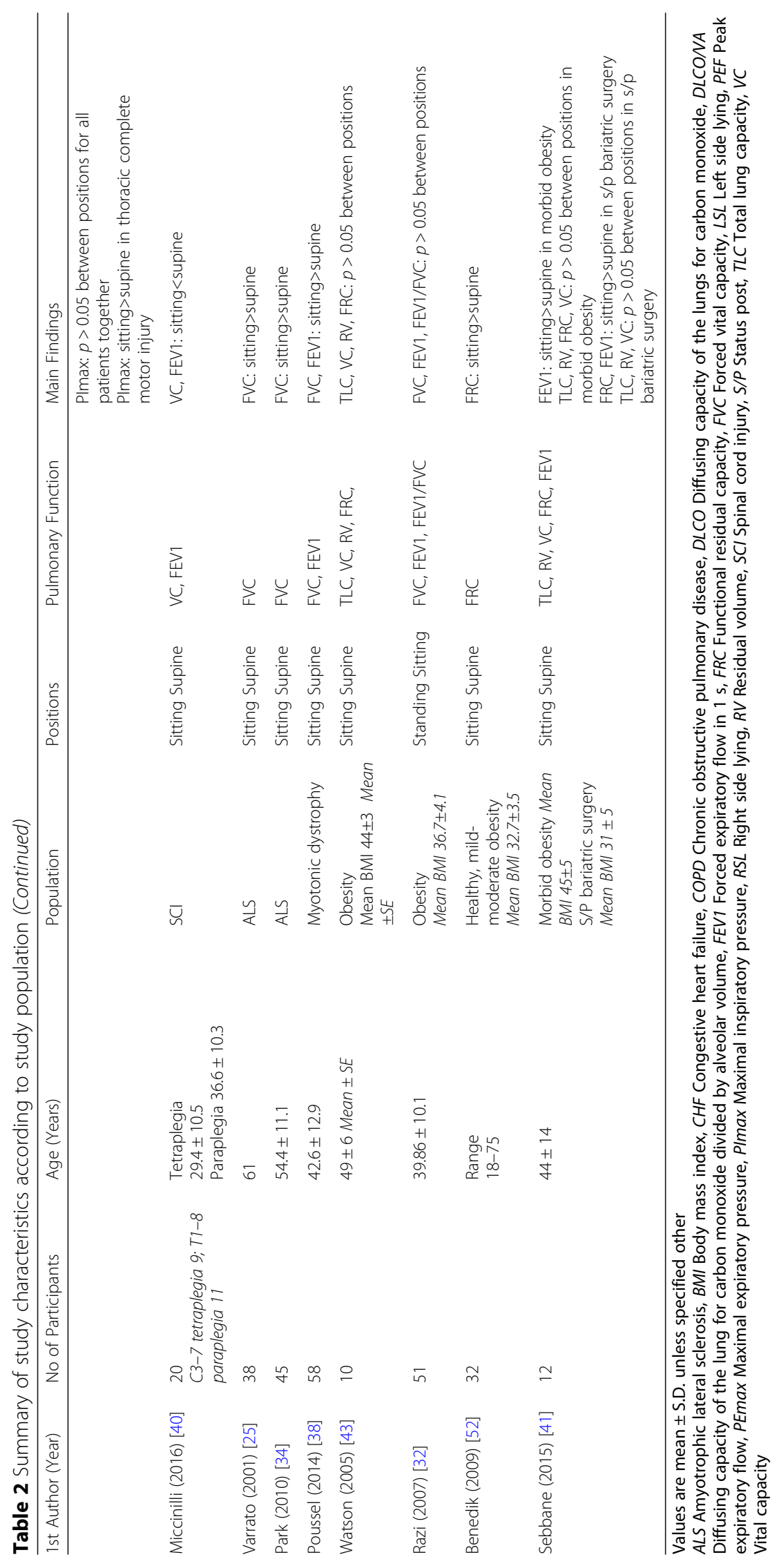


significance. Nevertheless, it is important to note that in these debilitated patients with SCI, even a small change in FVC is probably clinically significant.

Three studies evaluated patients with neuromuscular diseases [25, 34, 38]. In patients with myotonic dystrophy and in those with amyotrophic lateral sclerosis (ALS), there was a clinically and statistically significant decrease in FVC from sitting to supine [25, 34, 38]. In subjects with obesity (mean BMI 36.7) no significant difference was reported between standing and sitting [32].

\section{FEV 1}

In healthy subjects, FEV1 was reported to be higher in sitting vs. supine [3, 18, 22, 23, 26, 27, 39], in sitting vs. RSL and LSL [3, 19, 20], in standing vs. sitting [23], and in standing vs. sitting, supine, RSL, and LSL [19]. However, other studies $[21,24,28,40]$ did not find significant difference for FEV1 between sitting and supine, RSL, and LSL. One study [22] reported a decrease of $120 \mathrm{ml}$ in FEV1 from sitting to standing, which is statistically but not clinically significant.

Among asthmatic patients, FEV1 was higher in the standing vs. supine position, a statistically and clinically significant change; however, there was no significant difference between sitting vs. supine, RSL, and LSL positions [30]. Another study in asthmatic patients reported FEV1 to be higher in standing vs. sitting, supine, RSL, and LSL, and in sitting vs. supine, RSL and LSL [31]. Among obese asthmatic patients and those with COPD, there was no significant difference in FEV1 between standing and sitting $[29,32]$.

In subjects with $\mathrm{CHF}$, one study found a statistically and clinically significant increase in FEV1 in sitting vs. RSL and LSL, but no difference between sitting and supine [21], while two other studies reported higher FEV1 in sitting vs. supine $[18,27]$.

In patients with SCI, FEV1 was recently reported to increase from sitting to supine [40]; however, other studies found that the effect of position on FEV1 in those with SCI depends on the level and extent of injury. In one study among all subjects with SCI, FEV1 was not significantly influenced by moving from sitting to supine [35], but patients with cervical injuries showed a tendency for increased FEV1 in the supine vs. sitting position while those with thoracic injuries tended towards increased FEV1 in the sitting position. Along the same vein, another study [36] found an increase is FEV1 in the sitting vs. the supine position in patients with lumbar injury while FEV1 was higher in the supine position for those with cervical spine or thoracic injuries. Although the differences between positions were not statistically significant, the effect of level of injury was statistically and clinically significant.
In another study [33], FEV1 was higher in supine vs. sitting in patients with complete tetraplegia, while in patients with incomplete injury there was no significant difference between positions. Another group [37] reported no significant change in FEV1 between the sitting and supine positions for a pooled group of patients with SCI, but in the subgroup of patients with incomplete motor injury and in those with incomplete thoracic motor injury there was a decrease in the supine position.

In patients with myotonic dystrophy, FEV1 decreased from sitting to supine [38]. Among those with obesity, FEV1 was higher in sitting vs. supine both before and after bariatric surgery [41]. In another study among obese patients, there was no difference in FEV1 between standing and sitting [32].

\section{FEV1/FVC}

Seven studies compared FEV1/FVC for different body positions in healthy subjects [18, 19, 23, 24, 27, 28, 42]. In several studies, FEV1/FVC was reported to be higher in sitting vs. supine [23, 28], in sitting vs. LSL [19], and in standing vs. supine, RSL, and LSL [19]; however, FEV1/FVC was $>70 \%$ in all body positions so the difference was not clinically significant. Other studies found no difference between sitting and supine [18, 24, 27] or standing, sitting, and supine [42].

Among subjects with asthma, $\mathrm{CHF}$, and obesity no statistically significant difference in FEV1/FVC was found between the different body postures [18, 27, 32, 42].

\section{Vital capacity}

The effect of body position on vital capacity was evaluated in six studies of healthy subjects [21, 24, 28, 39, 43, $44]$. In most studies no difference was reported between sitting and supine $[21,24,28,43]$ or between sitting and RSL or LSL [21]. One study [39] found that VC was higher in the sitting vs. supine position. However, another study [44] found that VC was higher in the supine vs. sitting position, but only in females.

In patients with $\mathrm{CHF}, \mathrm{VC}$ was reported to be higher in sitting vs. supine in one study [27] while another study found no statistically significant difference between these positions [21]. In patients with spinal cord injury, VC was higher in the supine vs. sitting position [40]. In subjects with obesity, no difference in VC was reported between the sitting and supine positions [41, 43].

\section{PEF}

PEF in different body positions was evaluated in 13 studies [3, 22-24, 31, 33, 45-51]. Eight studies evaluated only healthy adults $[3,22-24,45,48,50,51]$, three evaluated healthy subjects and patients with COPD or asthma [31, 46, 49], one included adult cystic fibrosis patients [47], and one included subjects with SCI [33]. 
Nine studies that compared standing or sitting positions vs. supine or RSL and LSL found higher PEF in standing and sitting [3, 22-24, 31, 45-48]. Three of six studies comparing the standing and sitting positions found higher PEF in standing $[46,50,51]$ and one reported higher PEF in sitting [22]. However, it is most likely that none of the differences reported in PEF are clinically significant. In SCI patients with complete tetraplegia PEF was found to be $12 \%$ higher in the supine vs. sitting position [33].

\section{FRC}

FRC was evaluated using helium dilution in five studies [27, 41, 43, 52, 53]. Among healthy subjects, FRC was higher in standing [53] and in sitting [27, 43] vs. supine, with the differences reaching statistical and clinical significance. However, the difference in sitting vs. supine was not significant among patients with obesity (mean BMI 44-45) [41, 43] or CHF [27], and was higher in sitting vs. supine in patients after bariatric surgery (mean BMI 31) [41]. Another study [52] involving subjects with mild-to-moderate obesity (mean BMI 32), reported that FRC was significantly higher both statistically and clinically in sitting vs. supine.

\section{Total lung capacity}

Two studies that evaluated TLC using helium dilution in healthy subjects [43] and in subjects with obesity [41, 43] found no statistically significant difference between the sitting and supine positions.

\section{Residual volume}

Two studies that evaluated RV using helium dilution in healthy subjects [43] and those with obesity [41, 43] found no statistically significant difference between sitting and supine.

\section{PEmax}

Six studies investigated the association between body position and PEmax in healthy subjects [3, 28, 39, 46, $54,55]$. PEmax was higher in standing vs. supine, in standing vs. sitting and RSL, in sitting vs. supine [54], and in sitting vs. supine and RSL [46]; however, the differences reported in those studies were not clinically significant. Other studies found no difference in PEmax between sitting and supine [28, 39], or between sitting, supine, RSL, and LSL [3, 55].

In COPD patients, PEmax was higher in standing or sitting vs. supine or RSL [46], and was higher in standing and sitting vs. RSL in patients with cystic fibrosis [47]. The differences were not clinically significant.

In subjects with SCI, PEmax was significantly higher in sitting vs. supine for all subjects, and for patients with motor complete injury or incomplete cervical motor injury [37].

\section{PImax}

In healthy subjects, PImax was improved in sitting vs. supine in two studies [3, 54]. However, other studies found no difference in PImax in sitting vs. supine [28, 39 , 55], or sitting vs. RSL and LSL $[3,55]$. In subjects with chronic SCI, no significant change was seen in PImax between sitting and supine, with the exception of a subgroup of patients with complete thoracic motor paresis where there was statistically and clinically significant improvement in sitting [37].

\section{DLCO}

Seven studies evaluated the effect of body position on diffusion capacity; six included healthy subjects $[18,20$, $21,24,56,57]$, three included patients with CHF $[18,21$, 58], and one included COPD patients [57].

Among healthy subjects, two studies [24, 56] found statistically and clinically significant improvement in DLCO in supine vs. sitting and one [57] found a trend towards increased DLCO in supine vs. sitting, however this difference did not reach statistical significance. One study [18] found DLCO to be higher in the sitting vs. supine positions while another study found no difference in DLCO between these positions [21]. One study [21] reported higher DLCO in sitting vs. side lying while another study [20] found no difference between these positions. In COPD patients, no statistically significant change in DLCO was found between the sitting and the supine position [57].

Three studies investigated diffusion capacity in patients with CHF $[18,21,58]$. One study [58] found that postural changes from the supine to sitting positions induced different responses in diffusion capacity. In some patients diffusion capacity improved in the sitting position and others showed no change or a decline. On the average no statistically significant difference was found between the two positions. The authors attributed the difference in responses to variations in pulmonary circulation pressures. Another study [18] found no significant difference in diffusion capacity between the sitting and the supine positions. Side-lying was reported to reduce DLCO in comparison to sitting in the third study [21].

\section{Discussion}

Most studies in this systematic review of 43 papers evaluating the effect of body position on pulmonary function found that pulmonary function improved with more erect posture in both healthy subjects and those with lung disease, heart disease, neuromuscular diseases, and obesity. In patients with SCI, the effect is more complex and depends on the severity and level of injury. In contrast, diffusion capacity, as assessed by DLCO, increases in the supine position in healthy subjects while 
the effect in CHF patients is thought to depend upon pulmonary circulation pressure.

Decreased FVC in more recumbent positions may reflect both increased thoracic blood volume due to gravitational facilitation of venous return, which is more important in patients with heart failure, as well as cephalic displacement of the diaphragm due to abdominal pressure in the recumbent positions, which is more important in obese subjects [59]. In side-lying positions, even though only the dependent hemi-diaphragm is displaced, the effect on FVC appears to be similar to that observed in a supine position [59]. Other factors that may contribute to lower FVC values in side-lying positions include increased airway resistance and decreased lung compliance secondary to anatomical differences between the left and right lungs, as well as shifting of the mediastinal structures [20].

FEV1 was also higher in erect positions. Recumbent positions limit expiratory volumes and flow, which may reflect an increase in airway resistance, a decrease in elastic recoil of the lung, or decreased mechanical advantage of forced expiration, presumably affecting the large airways [20]. In asthmatic patients the increase in FVC while standing might be due to the increased diameter of the airways in this position [30].

In patients with CHF the lungs are stiff and heavy, and the heart is large and heavy, increasing the negative effects of lung-heart interdependence [60]. As cardiac dimension increases, lung volume, mechanical function, and diffusion capacity decrease $[61,62]$; thus, the heart weighs on the diaphragm while sitting and on one of the lungs while in a side-lying position. This influences the ability of the lungs to expand laterally but allows the diaphragm to descend and the lungs to expand inferiorly. In side-lying positions, the heart weighs on one lung, compressing both the airways and lung parenchyma, leading to a reduction in FEV1 and FVC due to airway compression [21]. Both elastic (reduced lung compliance) and resistive loads are simultaneously increased in the supine position in CHF patients [63].

Changes in FVC from the sitting to supine positions may reflect diaphragm strength/paralysis. FVC is thus an important clinical tool for assessment of diaphragmatic weakness in patients with neuromuscular diseases [64]. In patients with ALS, supine FVC is a test of diaphragmatic weakness [65] that predicts orthopnea [25] and prognosis for survival $[66,67]$. The American Academy of Neurology has concluded that in ALS patients, supine FVC is probably more effective than erect FVC in detecting diaphragm weakness and correlates better with symptoms of hypoventilation [68].

In patients with cervical SCI (tetraplegia), FVC and FEV1 increase in the supine vs. sitting position. The diaphragm increases its inspiratory excursion in the supine position because its muscle fibers are longer at end expiration, and they operate at a more effective point of their length-tension curve [69-71]. This mechanism is especially important in patients for whom the diaphragm is the main muscle for breathing, since their intercostal and abdominal muscles are inactive due to SCI.

FRC was reported to increase in upright positions in healthy subjects $[27,43,53]$ and in patients with mild-to-moderate obesity $[41,52]$. Changing from a supine to an upright position increases FRC due to reduced pulmonary blood volume and the descent of the diaphragm. This may change the point in which tidal breathing occurs in the volume-pressure curve, which leads to increased lung compliance, and thus an identical pressure change would produce a greater inspired volume if there is no change in respiratory drive [53]. However, among patients with CHF, no difference in FRC between sitting and supine was reported [27]. In heart failure, reduction in lung compliance in the supine position might reduce the passive change in lung volume, but FRC may be sustained above relaxation volume by an adjustment in respiratory muscle or glottal activity [27]. Among patients with obesity the sitting FRC was less than in healthy subjects but there was no further decrease in the supine position [43].

PEF, PEmax, and PImax were found to increase in upright positions in healthy subjects $[3,23,24,46,48,50$, 51] and in those with lung diseases [31, 46, 47]. This may be related to changes in lung volumes with positions.

Standing and sitting have been shown to lead to the highest lung volumes [72, 73]. At higher lung volumes the elastic recoil of the lungs and the chest wall is greater. In addition, the expiratory muscles are at a more optimal region of the length-tension curve and thus are capable of generating higher intrathoracic pressure, potentially generating higher expiratory pressures and pushing air through narrow airways at high speed, which results in higher PEmax, PEF, and FEV1. As lung volumes decrease, muscle length becomes less optimal, which results in lower PEmax in sitting, compared to the standing position, and even lower in more recumbent positions. The change in PEmax influences PEF [46].

When standing, gravity pulls the mediastinal and abdominal structures down, creating more space in the thoracic cavity, which allows further expansion of the lungs and greater lung volumes [74]. This, along with the decrease in compression on the lung bases, allows alveoli to recruit and increases lung compliance. The inspiratory muscles can expand even more, which allows the diaphragm to continue contracting downwards, thus increasing lung volumes [46].

Sitting often leads to the somewhat reduced lung volumes compared with standing. This can be explained by several mechanisms. First, in sitting, abdominal organs 
are higher, interfering with diaphragmatic motion, thus enabling smaller inspiration. Second, the abdominal muscles are in a less optimal point in the length-tension curve, since the combination of hip flexion and higher position of the abdominal contents exert upward pressure. Third, the back of the chair may limit thoracic expansion. These three factors explain a slightly lower PEmax and PEF in sitting vs. standing [46].

Diaphragmatic strength is negatively affected by the supine position, and intrathoracic blood volume is increased. These factors lead to decreased PEmax and PEF in the supine position [3].

In side-lying positions (RSL or LSL), when the bed is flat, the abdominal contents fall forward. The dependent hemi-diaphragm is stretched to a good length for tension generation, while the nondependent hemi-diaphragm is more flattened. Changes in lung volumes may thus balance themselves out due to a better diaphragmatic contraction but decreased space in the thorax [46].

The decreased PImax observed in the supine position could be related to diaphragm overload by abdominal content displacement during maximal inspiratory effort, which could offset improved diaphragm position on the length-tension curve. In addition, the length of all other inspiratory muscles may become less optimal in supine position [75].

In patients with cervical spinal cord injury and high tetraplegia, PEF was found to be higher in the supine vs. sitting position [33] corresponding to the increase in FVC and FEV1 in the supine position.

In healthy subjects, most studies showed an increase in DLCO in supine vs. sitting $[24,56,57]$. This improvement is attributed to the moderate increase in alveolar blood volume in the supine position due to recruitment of lung capillary bed on transition from upright to supine. Age may attenuate this increase [76]. This may explain why a study that included participants with a mean age of 61 [21] found no difference in DLCO between sitting and supine.

In side-lying positions, the heart weighs on one lung, compressing both airways and lung parenchyma, reducing alveolar blood volume, and causing ventilation/ perfusion mismatch. Those effects caused reduction of diffusion capacity in the side-lying positions [21].

In COPD patients, there was no change in DLCO between sitting and supine [57]. This might be related to reduced FVC and alveolar damage in these patients. These effects might have negative impact on diffusion capacity, opposing the positive effect of the increase in blood volume in the alveoli [57].

In patients with $\mathrm{CHF}$, different patterns of the effect of posture on DLCO were observed [58]. The change in DLCO was probably related to the change in alveolar blood volume, most likely due to differences in pulmonary artery pressure and heart dimensions [58].

\section{Limitations of the study}

There are a few limitations to this review. First, the level of evidence of the studies is relatively low. However, in this type of research, due to the nature of the populations studied and the interventions applied, it is impossible to perform a randomized control study. Second, most studies were performed on a small number of subjects and all studies used either consecutive, convenience, or volunteer sampling. The review included only adult subjects and it is therefore not possible to generalize the results to children and adolescents. Finally, research protocols varied between studies and detailed information about protocols were often missing. Patient cooperation during lung function testing strongly influences results. This may explain contradictory results obtained in some cases. Studies that included subjects older than 60 years did not mention the cognitive function of participants, a factor that may influence patient cooperation.

Further research in this field is needed, including studies designed to evaluate lung function in a larger number of healthy participants as well as in individuals with a variety of medical conditions. There is also a need to use a standardized protocol including randomization of postures and times between tests (e.g. for wash-out of inhaled gasses or redistribution of blood volume) in different positions to enable a better comparison of outcomes.

\section{Conclusions}

When performing pulmonary function tests, body position plays a role in its influence over test results. As seen in this review, a change in body position may have varying implications depending on the patient populations. American Thoracic Society (ATS) guidelines [2] recommend performing PFTs in the sitting or standing position, but the sitting position is usually preferred. The norms of those functions according to gender and age were established from tests performed in this position. This review suggests that for most of the subjects this is the preferred position for the test; however, clinicians should consider performing PFTs in other positions in selected patients. In patients with SCI, testing also in the supine position may provide important information. In patients with neuromuscular disorders, performing PFTs in the supine position may help to assess diaphragmatic function.

Positioning plays an important role in maximizing respiratory function when treating patients with various problems and diseases and it is important to know the implications of each position on the respiratory system of a specific patient. Understanding the influence of body position can give healthcare professionals better knowledge of optimal positions for patients with different diseases. 


\section{Additional files}

Additional file 1: Table S1. Scoring for papers included in the systematic review based on the Quality Assessment Tool for Before-After (Pre-Post) Studies with No Control Group of the National Heart, Lung and Blood Institute [3, 15-31, 33-58]. (DOCX 63 kb)

Additional file 2: Table S2. Statistically significant differences in pulmonary function between the various body positions $[3,17-28,30,31$, 33, 34, 37-41, 43-48, 50-54, 56]. (DOCX 104 kb)

\section{Abbreviations}

AAN: American Academy of Neurology; ALS: Amyotrophic lateral sclerosis; ATS: American Thoracic Society; CHF: Congestive heart failure; COPD: Chronic obstructive pulmonary disease; DLCO: Diffusing capacity of the lungs for carbon monoxide; ERS: European Respiratory Society; FEV1: Forced expiratory volume in $1 \mathrm{~s}$; FRC: Functional residual capacity; FVC: Forced vital capacity; LSL: Left side lying; PEF: Peak expiratory flow; PEmax: Maximal expiratory pressure; PFT: Pulmonary function test; PImax: Maximal inspiratory pressure; RSL: Right side lying; RV: Residual volume; SCl: Spinal cord injury; TLC: Total lung capacity; VC: Vital capacity

\section{Acknowledgements}

The authors wish to thank Prof. Ora Paltiel, a specialist in Internal Medicine, Hematology, and Oncology who also holds a doctorate in Epidemiology and Biostatistics, for her invaluable assistance in selecting the optimal tools for assessment of the quality of evidence and potential for bias of studies included in this systematic review.

The authors wish to thank Shifra Fraifeld, a medical center-based medical writer and editor, for her editorial contribution during manuscript preparation.

\section{Authors' contributions}

SK, E-LM, NA, AR contributed to the study concept and design. SK, E-LM, NA, $A R, Y Z$ contributed to data acquisition and analysis, and interpretation of the data. The primary literature search was conducted by SK and E-LM. SK and E-LM drafted the manuscript. SK, E-LM, NA, AR, YZ critically reviewed and revised the manuscript for intellectual content. All authors reviewed the final version of the manuscript prior to submission and all accept responsibility for the integrity of the research process and findings. All authors read and approved the final manuscript.

\section{Ethics approval and consent to participate}

Not applicable - systematic review.

\section{Consent for publication}

Not applicable.

\section{Competing interests}

The authors declare that they have no competing interests.

\section{Publisher's Note}

Springer Nature remains neutral with regard to jurisdictional claims in published maps and institutional affiliations.

\section{Author details}

${ }^{1}$ Chronic Ventilator-Dependent Division, Herzog Medical Center, POB 3900, Jerusalem, Israel. ${ }^{2}$ Pulmonary Institute, Shaare Zedek Medical Center, POB 3235, Jerusalem, Israel. ${ }^{3}$ Recanati School for Community Health Professions, Faculty of Health Sciences, Ben Gurion University of the Negev, Beer Sheva, Israel. ${ }^{4}$ Hebrew University-Hadassah Faculty of Medicine, Jerusalem, Israel.

Received: 6 May 2017 Accepted: 17 September 2018

Published online: 11 October 2018

\section{References}

1. Crapo RO. Pulmonary-function testing. N Engl J Med. 1994;331(1):25-30.

2. Miller MR, Crapo R, Hankinson J, et al. General considerations for lung function testing. Eur Respir J. 2005;26(1):153-61.
3. Meysman M, Vincken W. Effect of body posture on spirometric values and upper airway obstruction indices derived from the flow-volume loop in young nonobese subjects. Chest. 1998;114(4):1042-7.

4. Pellegrino R, Viegi G, Brusasco V, et al. Interpretative strategies for lung function tests. Eur Respir J. 2005;26(5):948-68.

5. Wanger J, Clausen $J$, Coates $A$, et al. Standardisation of the measurement of lung volumes. Eur Respir J. 2005;26(3):511-22.

6. Goswami R, Guleria R, Gupta AK, et al. Prevalence of diaphragmatic muscle weakness and dyspnoea in Graves' disease and their reversibility with carbimazole therapy. Eur J Endocrinol. 2002;147(3):299-303.

7. Keenan SP, Alexander D, Road JD, Ryan CF, Oger J, Wilcox PG. Ventilatory muscle strength and endurance in myasthenia gravis. Eur Respir J. 1995;8(7): $1130-5$

8. Nava S, Crotti P, Gurrieri G, Fracchia C, Rampulla C. Effect of a beta 2 agonist (broxaterol) on respiratory muscle strength and endurance in patients with COPD with irreversible airway obstruction. Chest. 1992; 101(1):133-40.

9. Quanjer PH, Lebowitz MD, Gregg I, Miller MR, Pedersen OF. Peak expiratory flow: conclusions and recommendations of a working Party of the European Respiratory Society. Eur Respir J Suppl. 1997;24:2s-8s.

10. Global initiative for asthma (GINA): Global strategy for asthma management and prevention (2018 update). 2018. file:///C:/Users/owner/Downloads/ wms-GINA-2018-report-V1.3-002.pdf. Accessed 29 May 2018.

11. Graham BL, Brusasco V, Burgos F, et al. 2017 ERS/ATS standards for singlebreath carbon monoxide uptake in the lung. Eur Respir J. 2017:49(1). https://doi.org/10.1183/13993003.00016-2016.

12. Hathaway EH, Tashkin DP, Simmons MS. Intraindividual variability in serial measurements of DLCO and alveolar volume over one year in eight healthy subjects using three independent measuring systems. Am Rev Respir Dis. 1989:140(6):1818-22.

13. Moher D, Liberati A, Tetzlaff J, Altman DG, PRISMA Group. Preferred reporting items for systematic reviews and meta-analyses: the PRISMA statement. PLoS Med. 2009;6(7):e1000097.

14. Gronseth GS, Woodroffe LM, Getchuis TSD. Clinical practice guideline process manual. 2011. http://tools.aan.com/globals/axon/assets/9023.pdf. Accessed 29 May 2018.

15. Quality assessment tool for before-after (pre-post) studies with no control group. 2014. https://www.nhlbi.nih.gov/health-topics/study-qualityassessment-tools. Accessed 12 Aug 2018.

16. Kunstler BE, Cook JL, Freene N, et al. Physiotherapist-led physical activity interventions are efficacious at increasing physical activity levels: a systematic review and meta-analysis. Clin J Sport Med. 2018;28(3):304-15.

17. Ben-Dov I, Zlobinski R, Segel MJ, Gaides M, Shulimzon T, Zeilig G. Ventilatory response to hypercapnia in C(5-8) chronic tetraplegia: the effect of posture. Arch Phys Med Rehabil. 2009;90(8):1414-7.

18. Ceridon ML, Morris NR, Olson TP, Lalande S, Johnson BD. Effect of supine posture on airway blood flow and pulmonary function in stable heart failure. Respir Physiol Neurobiol. 2011;178(2):269-74.

19. Ganapathi LV, Vinoth S. The estimation of pulmonary functions in various body postures in normal subjects. Int J Advances Med. 2015;2(3):250-4 http://www. ijmedicine.com/index.php/ijam/article/view/360. Accessed 29 May 2018.

20. Manning F, Dean E, Ross J, Abboud RT. Effects of side lying on lung function in older individuals. Phys Ther. 1999:79(5):456-66.

21. Palermo P, Cattadori G, Bussotti M, Apostolo A, Contini M, Agostoni P. Lateral decubitus position generates discomfort and worsens lung function in chronic heart failure. Chest. 2005;128(3):1511-6.

22. Patel AK, Thakar HM. Spirometric values in sitting, standing, and supine position. Lung Pulm Resp Res. 2015;2(1):00026 http://medcraveonline.com/ JLPRR/JLPRR-02-00026.php. Accessed 29 May 2018.

23. Saxena J, Gupta S, Saxena S. A study of change of posture on the pulmonary function tests : can it help COPD patients? Indian J Community Health. 2006;18(1):10-2. http://www.iapsmupuk.org/journal/index.php/IJCH/ article/view/108. Accessed 29 May 2018

24. Stewart IB, Potts JE, McKenzie DC, Coutts KD. Effect of body position on measurements of diffusion capacity after exercise. Br J Sports Med. 2000; 34(6):440-4.

25. Varrato J, Siderowf A, Damiano P, Gregory S, Feinberg D, McCluskey L. Postural change of forced vital capacity predicts some respiratory symptoms in ALS. Neurology. 2001;57(2):357-9.

26. Vilke GM, Chan TC, Neuman T, Clausen JL. Spirometry in normal subjects in sitting, prone, and supine positions. Respir Care. 2000;45(4):407-10. 
27. Yap JC, Moore DM, Cleland JG, Pride NB. Effect of supine posture on respiratory mechanics in chronic left ventricular failure. Am J Respir Crit Care Med. 2000;162(4 Pt 1):1285-91.

28. Tsubaki A, Deguchi S, Yoneda Y. Influence of posture on respiratory function and respiratory muscle strength in normal subjects. J Phys Ther Sci. 2009;21(1):71-4 https://www.jstage.jst.go.jp/article/jpts/21/1/21_1_71/_ article. Accessed 29 May 2018.

29. De S. Comparison of spirometric values in sitting versus standing position among patients with obstructive lung function. Indian J Allergy Asthma Immunol. 2012;26(2):86-8 http://medind.nic.in/iac/t12/i2/iact12i2p86.pdf. Accessed 29 May 2018

30. Melam GR, Buragadda S, Alhusaini A, Alghamdi MA, Alghamdi MS, Kaushal P. Effect of different positions on FVC and FEV1 measurements of asthmatic patients. J Phys Ther Sci. 2014;26(4):591-3.

31. Mohammed J, Abdulateef A, Shittu A, Sumaila FG. Effect of different body positioning on lung function variables among patients with bronchial asthma. Arch Physiother Global Res. 2017;21(3):7-12. http://apgr.wssp.edu. pl/wp-content/uploads/2017/12/APGR-21-3-A.pdf. Accessed 29 May 2018.

32. Razi E, Moosavi GA. The effect of positions on spirometric values in obese asthmatic patients. Iran J Allergy Asthma Immunol. 2007;6(3):151-4.

33. Linn WS, Adkins RH, Gong H Jr, Waters RL. Pulmonary function in chronic spinal cord injury: a cross-sectional survey of 222 southern California adult outpatients. Arch Phys Med Rehabil. 2000;81(6):757-63.

34. Park JH, Kang SW, Lee SC, Choi WA, Kim DH. How respiratory muscle strength correlates with cough capacity in patients with respiratory muscle weakness. Yonsei Med J. 2010;51(3):392-7.

35. Baydur A, Adkins RH, Milic-Emili J. Lung mechanics in individuals with spinal cord injury: effects of injury level and posture. J Appl Physiol. 2001;90(2):405-11.

36. Kim M-K, Hwangbo $\mathrm{G}$. The effect of position on measured lung function in patients with spinal cord injury. J Physical Therapy Sci. 2012;24(8):655-7 https://www.jstage.jst.go.jp/article/jpts/24/8/24_JPTS-2012-029/_article. Accessed 29 May 2018.

37. Terson de Paleville DG, Sayenko DG, Aslan SC, Folz RJ, McKay WB, Ovechkin AV. Respiratory motor function in seated and supine positions in individuals with chronic spinal cord injury. Respir Physiol Neurobiol. 2014;203:9-14.

38. Poussel M, Kaminsky P, Renaud P, Laroppe J, Pruna L, Chenuel B. Supine changes in lung function correlate with chronic respiratory failure in myotonic dystrophy patients. Respir Physiol Neurobiol. 2014;193:43-51.

39. Naitoh S, Tomita K, Sakai K, Yamasaki A, Kawasaki Y, Shimizu E. The effect of body position on pulmonary function, chest wall motion, and discomfort in young healthy participants. J Manipulative Physiol Ther. 2014;37(9):719-25.

40. Miccinilli S, Morrone M, Bastianini F, et al. Optoelectronic plethysmography to evaluate the effect of posture on breathing kinematics in spinal cord injury: a cross sectional study. Eur J Phys Rehabil Med. 2016;52(1):36-47.

41. Sebbane M, El Kamel M, Millot A, et al. Effect of weight loss on postural changes in pulmonary function in obese dubjects: a longitudinal study. Respir Care. 2015;60(7):992-9.

42. Myint WW, Htay MNN, Soe HHK, et al. Effect of body positions on lungs volume in asthmatic patients: a cross-sectinal study. J Adv Med Pharma Sci. 2017;13(4):1-6 http://www.journalrepository.org/media/journals/JAMPS_36/ 2017/Jun/Myint1342017JAMPS33901.pdf. Accessed 29 May 2018.

43. Watson RA, Pride NB. Postural changes in lung volumes and respiratory resistance in subjects with obesity. J Appl Physiol (1985). 2005;98(2):512-7.

44. Roychowdhury P, Pramanik T, Prajapati R, Pandit R, Singh S. In health--vital capacity is maximum in supine position. Nepal Med Coll J. 2011;13(2):131-2.

45. Antunes BO, de Souza HC, Gianinis HH, Passarelli-Amaro RC, Tambascio J, Gastaldi AC. Peak expiratory flow in healthy, young, non-active subjects in seated, supine, and prone postures. Physiother Theory Pract. 2016;32(6):489-93.

46. Badr C, Elkins MR, Ellis ER. The effect of body position on maximal expiratory pressure and flow. Aust J Physiother. 2002:48(2):95-102

47. Elkins MR, Alison JA, Bye PT. Effect of body position on maximal expiratory pressure and flow in adults with cystic fibrosis. Pediatr Pulmonol. 2005;40(5):385-91.

48. Gianinis HH, Antunes BO, Passarelli RC, Souza HC, Gastaldi AC. Effects of dorsal and lateral decubitus on peak expiratory flow in healthy subjects. Braz J Phys Ther. 2013;17(5):435-41.

49. McCoy EK, Thomas JL, Sowell RS, et al. An evaluation of peak expiratory flow monitoring: a comparison of sitting versus standing measurements. J Am Board Fam Med. 2010;23(2):166-70.

50. Ottaviano G, Scadding GK, lacono V, Scarpa B, Martini A, Lund VJ. Peak nasal inspiratory flow and peak expiratory flow. Upright and sitting values in an adult population. Rhinology. 2016;54(2):160-3.
51. Wallace JL, George CM, Tolley EA, et al. Peak expiratory flow in bed? A comparison of 3 positions. Respir Care. 2013;58(3):494-7.

52. Benedik PS, Baun MM, Keus $L$, et al. Effects of body position on resting lung volume in overweight and mildly to moderately obese subjects. Respir Care. 2009;54(3):334-9.

53. Chang AT, Boots RJ, Brown MG, Paratz JD, Hodges PW. Ventilatory changes following head-up tilt and standing in healthy subjects. Eur J Appl Physiol. 2005;95(5-6):409-17.

54. Costa R, Almeida N, Ribeiro F. Body position influences the maximum inspiratory and expiratory mouth pressures of young healthy subjects. Physiotherapy. 2015;101(2):239-41.

55. Ogiwara S, Miyachi T. Effect of posture on ventilatory muscle strength. J Phys Ther Sci. 2002;14(1):1-5. https://www.jstage.jst.go.jp/article/jpts/14/1/ 14_1_1/_pdf/-char/en. Accessed 29 May 2018.

56. Peces-Barba G, Rodriguez-Nieto MJ, Verbanck S, Paiva M, Gonzalez-Mangado N. Lower pulmonary diffusing capacity in the prone vs. supine posture. J Appl Physiol (1985). 2004;96(5):1937-42.

57. Terzano C, Conti V, Petroianni A, Ceccarelli D, De Vito C, Villari P. Effect of postural variations on carbon monoxide diffusing capacity in healthy subjects and patients with chronic obstructive pulmonary disease. Respiration. 2009;77(1):51-7.

58. Faggiano $P$, D'Aloia A, Simoni $P$, et al. Effects of body position on the carbon monoxide diffusing capacity in patients with chronic heart failure: relation to hemodynamic changes. Cardiology. 1998;89(1):1-7.

59. Behrakis PK, Baydur A, Jaeger MJ, Milic-Emili J. Lung mechanics in sitting and horizontal body positions. Chest. 1983;83(4):643-6.

60. Agostoni PG, Marenzi GC, Sganzerla P, et al. Lung-heart interaction as a substrate for the improvement in exercise capacity after body fluid volume depletion in moderate congestive heart failure. Am J Cardiol. 1995;76(11):793-8.

61. Agostoni PG, Cattadori G, Guazzi M, Palermo P, Bussotti M, Marenzi G. Cardiomegaly as a possible cause of lung dysfunction in patients with heart failure. Am Heart J. 2000;140(5):e24

62. Hosenpud JD, Stibolt TA, Atwal K, Shelley D. Abnormal pulmonary function specifically related to congestive heart failure: comparison of patients before and after cardiac transplantation. Am J Med. 1990;88(5):493-6.

63. Nava S, Larovere MT, Fanfulla F, Navalesi P, Delmastro M, Mortara A. Orthopnea and inspiratory effort in chronic heart failure patients. Respir Med. 2003;97(6):647-53.

64. Fromageot $C$, Lofaso $F$, Annane $D$, et al. Supine fall in lung volumes in the assessment of diaphragmatic weakness in neuromuscular disorders. Arch Phys Med Rehabil. 2001;82(1):123-8.

65. Lechtzin N, Wiener CM, Shade DM, Clawson L, Diette GB. Spirometry in the supine position improves the detection of diaphragmatic weakness in patients with amyotrophic lateral sclerosis. Chest. 2002;121(2):436-42.

66. Baumann F, Henderson RD, Morrison SC, et al. Use of respiratory function tests to predict survival in amyotrophic lateral sclerosis. Amyotroph Lateral Scler. 2010;11(1-2):194-202.

67. Schmidt EP, Drachman DB, Wiener CM, Clawson L, Kimball R, Lechtzin N. Pulmonary predictors of survival in amyotrophic lateral sclerosis: use in clinical trial design. Muscle Nerve. 2006;33(1):127-32.

68. Miller RG, Jackson CE, Kasarskis EJ, et al. Practice parameter update: the care of the patient with amyotrophic lateral sclerosis: drug, nutritional, and respiratory therapies (an evidence-based review): report of the quality standards Subcommittee of the American Academy of Neurology. Neurology. 2009;73(15):1218-26.

69. Fugl-Meyer AR. Effects of respiratory muscle paralysis in tetraplegic and paraplegic patients. Scand J Rehabil Med. 1971;3(4):141-50.

70. Fugl-Meyer AR, Grimby G. Respiration in tetraplegia and in hemiplegia: a review. Int Rehabil Med. 1984:6(4):186-90.

71. Huldtgren AC, Fugl-Meyer AR, Jonasson E, Bake B. Ventilatory dysfunction and respiratory rehabilitation in post-traumatic quadriplegia. Eur J Respir Dis. 1980;61(6):347-56

72. Wade OL, Gilson JC. The effect of posture on diaphragmatic movement and vital capacity in normal subjects with a note on spirometry as an aid in determining radiological chest volumes. Thorax. 1951;6(2):103-26.

73. Moreno F, Lyons HA. Effect of body posture on lung volumes. J Appl Physiol. 1961;16:27-9.

74. Castile R, Mead J, Jackson A, Wohl ME, Stokes D. Effects of posture on flowvolume curve configuration in normal humans. J Appl Physiol Respir Environ Exerc Physiol. 1982;53(5):1175-83. 
75. Segizbaeva MO, Pogodin MA, Aleksandrova NP. Effects of body positions on respiratory muscle activation during maximal inspiratory maneuvers. Adv Exp Med Biol. 2013;756:355-63.

76. Chang SC, Chang HI, Liu SY, Shiao GM, Perng RP. Effects of body position and age on membrane diffusing capacity and pulmonary capillary blood volume. Chest. 1992;102(1):139-42.

Ready to submit your research? Choose BMC and benefit from:

- fast, convenient online submission

- thorough peer review by experienced researchers in your field

- rapid publication on acceptance

- support for research data, including large and complex data types

- gold Open Access which fosters wider collaboration and increased citations

- maximum visibility for your research: over $100 \mathrm{M}$ website views per year

At $\mathrm{BMC}$, research is always in progress.

Learn more biomedcentral.com/submissions 